\title{
Verbesserte Prognose bei Prostatakarzinom mit Gleason-Score 6 oder 7
}

\author{
Durch die Modifikation des Gleason-Gradings im Jahr 2005 ist bei \\ einigen Prostatakarzinomen der Punktwert von 6 auf 7 (3+4) hochgestuft \\ worden. Beide Gruppen haben dadurch heute eine bessere Prognose als \\ zuvor.
}

—ür die feingewebliche Beurteilung ei- nes Prostatakarzinoms mittels Gleason-Score (GS) werden seit der Neufassung im Jahr 2005 nicht mehr die beiden häufigsten, sondern der häufigste und der höchste Gleason-Grad addiert. Außerdem wurde vor elf Jahren die Definition von Grad 3 und 4 so abgewandelt, dass bestimmte Muster des vormaligen Grades 3 nun als Grad 4 klassifiziert werden. Einige Prostatakarzinome, die früher als GS 6 eingestuft worden wären, gelten damit heute als GS 7 $(3+4)$. Dänische Urologen geben mit ihrer in diesem Jahr veröffentlichten Studie nun zu bedenken, dass diese Änderungen eine Überarbeitung der Therapieempfehlungen notwendig machen könnte.

Wie die Ärzte um Kasper D. Berg von der Universität Kopenhagen zeigen konnten, haben Patienten sowohl mit GS-6- als auch mit GS-7-Tumoren heute nach einer radikalen Prostatektomie ein deutlich geringeres Risiko für ein biochemisches Rezidiv als vor der Revision des Tumorgradings durch die ISUP (International Society of Urological Pathology).

Für ihren paarweisen Vergleich verwendeten Berg und Kollegen prospektiv an ihrer Klinik erhobene Daten. 215 Prostatakarzinompatienten, die sich vor Ende 2005 einer radikalen Prostatektomie (RP) unterzogen hatten (Prä-ISUP), wurden ebenso vielen Patienten mit einer RP zwischen 2008 und 2011 (PostISUP) gegenübergestellt. In beiden Gruppen hatten jeweils 157 Männer ein GS-6- und 58 Männer ein GS-7(3+4)Karzinom. Die Patientenpaare waren so gewählt, dass sie außer im GS auch in Alter, klinischem Tumorstadium, PSAWert und postoperativem Randstatus übereinstimmten.

Die Fünf-Jahres-Inzidenz an biochemischen Rezidiven, definiert als An-

\section{Hier steht eine Anzeige.}

stieg des PSA-Wertes $\geq 0,2 \mathrm{ng} / \mathrm{ml}$, betrug $34,0 \%$ in der Prä- und 13,9 \% in der Post-ISUP-Gruppe. Die Reduktion der Rezidivrate betraf beide GS-Gruppen: Bei einem GS von 6 war sie von $29,3 \%$ (Prä-ISUP) auf 11,2 \% (PostISUP) und bei einem GS von $7(3+4)$ von $46,6 \%$ auf $21,1 \%$ zurückgegangen. Post-ISUP-Patienten mit GS $7(3+4)$ hatten also eine vergleichbare BR-Rate wie Prä-ISUP-Patienten mit GS 6. Die Neufassung des GS-Gradings erwies sich damit als unabhängiger Marker für ein geringeres Risiko an biochemischen Rezidiven.

Fazit: Laut Berg und seinen Kollegen ist die GS-6-Gruppe heute „eine homogenere Gruppe als in der Prä-ISUP-Ära mit einer exzellenten Prognose“. Gleichzeitig sei durch das modifizierte Grading die prognostische und klinische Aussagekraft von GS 7 (3+4) - wo 1-49\% der Tumorzellen als Grad 4 klassifiziert sein können - geschwächt worden.

„Durch eine Verdünnung der Gruppe durch Patienten, die früher als GS 6 klassifiziert wurden, scheint sich die Prognose von GS-7-Patienten verbessert zu haben“, so Berg und Kollegen. Studienergebnisse mit dem alten GSSystem seien daher nicht exakt auf jüngere Kohorten übertragbar. Das habe auch Konsequenzen für therapeutische Entscheidungen. So werden in Programmen zur aktiven Überwachung derzeit meist keine Patienten mit einem $\mathrm{GS} \geq 7$ einbezogen. Vor allem für GS-3+4-Patienten mit minimalem Grad-4-Anteil könnte laut Berg und Kollegen eine sofortige RP aber eine Übertherapie darstellen.

Dr. Beate Schumacher

Berg KD et al. The impact of the 2005 International Society of Urological Pathology consensus guidelines on gleason grading - a matched pair analysis. BJU Int. 2016;doi:10.1111/bju.13439. 